\title{
Editorial: Microbiological Risks in Food Processing
}

\author{
Paula Teixeira ${ }^{1}$, Avelino Alvarez-Ordóñez ${ }^{2}$ and Guadalupe Virginia Nevárez-Moorillón ${ }^{3 *}$ \\ ${ }^{1}$ CBQF-Centro de Biotecnologia e Química Fina-Laboratório Associado, Universidade Católica Portuguesa, Escola \\ Superior de Biotecnologia, Porto, Portugal, ${ }^{2}$ Department of Food Hygiene and Technology and Institute of Food Science and \\ Technology, Universidad de León, León, Spain, ${ }^{3}$ Facultad de Ciencias Químicas, Universidad Autónoma de Chihuahua, \\ Chihuahua, Mexico
}

Keywords: foodborne disease, microbiological risk, sustainable food processing, pathogens, environmental sustainability, food matrix, innovative technologies

\section{Editorial on the Research Topic}

\section{Microbiological Risks in Food Processing}

Risks associated with food as a vehicle for transmitting pathogens are of utmost importance to provide safe and nutritious food for consumers worldwide. Foodborne diseases cause significant morbidity and mortality and can be a barrier to socio-economic development, considering the number of infectious diseases related to food transmission, higher in low-income regions. Therefore, food safety is a key element to contribute to the achievement of the Sustainable Development Goals 2.0 (Zero Hunger), 3.0 (Good Health and Well-being), 6.0 (Clean Water and Sanitation), and 12.0 (Responsible Production and Consumption).

Food processing methodologies are directed to control microbial growth or inactivate microorganisms in food products, to lower the risk of foodborne pathogens or spoilage microorganisms. Control of microorganisms also needs to include the equipment and surfaces of the processing plant. On the other hand, for the addition of antimicrobial compounds to product formulation, the effect of other food components or food processing needs to be considered. As for foodborne pathogens, the availability of methods for isolation, identification, and molecular typing, can provide important information on the dispersion of pathogens and the related outbreaks.

Therefore, the microbial interactions with food products and processes need to be analyzed at all stages of the food production chains, from farm to fork. The articles included in this Research Topic analyse the use of sustainable disinfectants in the food industry, the presence of foodborne pathogens in farms, and the use of biocontrol strategies in food formulation.

The microorganisms present in food processing plants can be a source of microbial spoilers and foodborne pathogens that can contaminate the processed food products, increasing the microbiological risks associated to the specific food product. In Feye et al., the University of Arkansas group in collaboration with the Research Center from the United States Department of Agriculture present the results of the comparison of two sanitizers. Peracetic acid and sodium bisulfate were used to sanitize re-used water from a poultry facility. Although both sanitizers were effective in decreasing the microbial load in the re-used water, a difference in the remaining microbiome was observed; therefore, it is important to select sanitizers used in the food industry properly. In López-Gálvez et al., the use of chlorine dioxide in a tomato packinghouse is revised to assess the sanitizer's efficiency in the water used in the process to consider its re-use. This is a contribution from CEBAS-CSIC, Murcia, Spain. The results demonstrated that the use of chlorine dioxide allowed the re-use of water, with a similar effect on the tomato washing process as when tap water was used; the by-products of the sanitizer used were not detected. Although the use 
of disinfectants can help control microorganisms in the processing plants, good manufacturing practices need to be monitored to assure the processing of safe foods.

The identification and characterization of pathogenic microorganisms can also help characterize microbial risks associated with animal and plant-based foods. In the research group's contribution from Australia and United Kingdom (Bean et al.), the methodology for isolation and characterization of polymyxin-resistant Gram-negative pathogens in a poultry farm is presented. The samples were taken at different facilities in Australia, and the isolates were identified and characterized for their antibiotic resistance and the presence of colistin resistance genes. An important conclusion is that there was a high proportion of isolates with antibiotic resistance and highlights the importance of constant surveillance on drug-resistant strains along the food production process. The importance of a particular pathogen in food processing systems, such as Listeria monocytogenes (Stone et al.), is emphasized due to its adaptability to different conditions and its ubiquitous presence in the environment. The report by the group from Ireland, France, and Germany, proposed the use of a lytic bacteriophage specific for L. monocytogenes, to control the pathogen's growth in ready-to-eat meals artificially contaminated. Bacteriophages are a novel approach for the control of pathogens in food but can be a novel tool for decreasing the risks associated with specific pathogens in a particular food product.

The effect of the essential oil obtained from Mexican oregano (Lippia berlandieri Schauer) on the control of Pseudomonas aeruginosa and Salmonella Typhimurium biofilm formation on stainless steel surfaces is analyzed by the group of University of Puebla, Mexico (Reyes-Jurado et al.). Modeling tools were used to determine the effect of a layer of the essential oil on the stainless steel surface, which demonstrated an inhibition on biofilm formation or a delay on the biofilm's initial formation; the effect was different for the two pathogens studied. The interaction of food components with the antifungal effect of the Mexican oregano essential oil (Levario-Gómez et al.) is presented in another contribution by the University of Chihuahua in Mexico, in collaboration with Puebla and the University of the Americas in Puebla. The concentration of proteins, the matrix $\mathrm{pH}$, and the concentration of the Mexican oregano essential oil was analyzed on the growth of two food spoilage microorganisms, and predictive models were used to assess the combination of effects on fungal growth. The differences in the concentration of the bioactive components in the Mexican oregano essential oil also affected their antifungal capacity. In the previous two contributions, predictive microbiology is presented in the analysis of microbial risks in food production systems.

In another contribution to the Research Topic, Salgado-Nava et al. present the effect of the addition of encapsulated Mexican oregano essential oil to bagels that were artificially contaminated with fungal strains. The contribution is presented by the group from the University of the Americas in Puebla. Even when the addition of the emulsion on the bagel's surface did not affect the bread pieces' physicochemical properties, there was an antifungal effect observed that delayed the fungal growth for several days.

Food products' safety is as important as their nutritious quality, and it is necessary to identify and control the conditions that can alter the microbiological quality of foods. From the presence of pathogenic microorganisms in animals and crops to food processing plants and food formulation, the microbiological risks associated with food processing and distribution need to be carefully monitored.

\section{AUTHOR CONTRIBUTIONS}

All authors listed have made a substantial, direct and intellectual contribution to the work, and approved it for publication.

\section{ACKNOWLEDGMENTS}

We thank Frontiers for all their support.

Conflict of Interest: The authors declare that the research was conducted in the absence of any commercial or financial relationships that could be construed as a potential conflict of interest.

Copyright (c) 2021 Teixeira, Alvarez-Ordónez and Nevárez-Moorillón. This is an open-access article distributed under the terms of the Creative Commons Attribution License (CC BY). The use, distribution or reproduction in other forums is permitted, provided the original author(s) and the copyright owner(s) are credited and that the original publication in this journal is cited, in accordance with accepted academic practice. No use, distribution or reproduction is permitted which does not comply with these terms. 